$56^{\text {ème }}$ Congrès de la SFMBCB, 01002 (2011)

DOI: $10.1051 / \mathrm{sfmbcb} / 20115601002$

(C) Owned by the authors, published by EDP Sciences, 2011

\title{
Comment le passage à la 3D va-t-il modifier nos diagnostics et nos thérapeutiques ?
}

\author{
Cavézian R, Pasquet G
}

\author{
Cabinet de Radiologie dentaire Echelle-Saint Honoré \\ echellesthonore@yahoo.fr
}

L'imagerie de projection, principe initial de l'image radiologique (1895), réduit un volume anatomique $3 \mathrm{D}$ en une image plane 2D. Le souci de la troisième dimension axiale va conduire, par des procédés toujours plus complexes et performants, à une imagerie enfin 3D.

La première étape est la tomographie conventionnelle (1922) qui discrimine, plan par plan, les structures complexes selon, au mieux, deux orientations orthogonales (frontale et sagittale).

La deuxième étape, celle du scanner Rx (1972) allie faisceau collimaté et numérisation. Le volume anatomique est obtenu par la superposition des coupes d'acquisition. Les reconstructions 2D et 3D volumiques sont réalisées par l'outil informatique (1985). Dans les années 1990, les Directives européennes de radioprotection obligent les radiologues à optimiser les examens (protocoles « low dose » du scanner) et induisent le développement du Cone Beam.

La troisième étape, celle du Cone Beam $(\mathrm{CB} / \mathrm{CBCT})$ reprend le principe géométrique initial. Par un nombre fini de projections multi-angulaires 2D de l'objet sur un capteur numérique, on obtient le volume «voxélisé » de l'objet. Spécifique de l'évaluation des structures denses (os, dent...), mais insuffisant pour les parties molles, moins irradiant que le scanner même optimisé, de résolution au moins égale à ce dernier avec les appareils haut de gamme, le CB est aujourd'hui la technique de référence en imagerie dento-maxillo-faciale. Les reconstructions planes ou courbes 2D et de rendu 3D sont réalisées par des logiciels dédiés.

La simulation et la robotique exploitent les données d'acquisition. Par son atout dosimétrique, le CB est prescrit, avec moins de réticence que le scanner, dans l'imagerie 3D de l'enfant.

Comme pour le scanner Rx, l'implantologie a été la première application du Cone Beam (imagerie sectionnelle, simulation, robotique..) et a rapidement gagné les autres secteurs de l'odontologie et de la chirurgie maxillo-faciale :

- la localisation des apex des 3èmes molaires mandibulaires constitue une première extension;

- la possibilité d'une exploration 3D représente un élément déterminant dans le diagnostic des fissures radiculaires et des lésions parodontales associées, par l'utilisation de champs réduits;

- l'orthopédie dento-faciale et la chirurgie maxillo-faciale (éléments dentaires inclus, dysraphies, céphalométrie 3D, malformations faciales ...) bénéficient aussi du Cone Beam;

- l'étude des cavités sinusiennes et des composantes osseuses des ATM également;

- l'évaluation des processus tumoraux est aisément réalisée. Toutefois, les lésions propagées aux parties molles relèvent du scanner et/ou de l'IRM.

Par ses qualités, le Cone Beam est un instrument diagnostique affuté. Son utilisation réclame une compétence radio-clinique particulière. Reprenant les propositions de l'Académie européenne de Radiologie dentaire et maxillo-faciale, la Haute Autorité de Santé se prononce pour un enseignement diplômant pluridiciplinaire spécifique au Cone Beam. 